\title{
Efficacy of a three-day prolonged-course of multiple-dose versus a single-dose of tranexamic acid in total hip and knee arthroplasty
}

\author{
Xiang-Dong $\mathrm{Wu}^{1,2}$, Mian Tian ${ }^{3}$, Yao $\mathrm{He}^{4}$, Yu Chen ${ }^{5}$, Yu-Zhang Tao $^{2}$, Long Shao ${ }^{6}$, Changqi Luo ${ }^{2}$, \\ Peng-Cheng Xiao ${ }^{2}$, Zheng-Lin Zhu ${ }^{2}$, Jia-Cheng Liu ${ }^{2}$, Wei Huang ${ }^{2}$, Gui-Xing Qiu ${ }^{1}$
}

${ }^{1}$ Department of Orthopaedic Surgery, Peking Union Medical College Hospital, Chinese Academy of Medical Sciences \& Peking Union Medical College, Beijing 100730, China; ${ }^{2}$ Department of Orthopaedic Surgery, The First Affiliated Hospital of Chongqing Medical University, Chongqing 400016, China; ${ }^{3}$ Department of Orthopaedic Surgery, Dianjiang People's Hospital, Chongqing 408300, China; ${ }^{4}$ Department of Orthopaedic Surgery, Banan People's Hospital of Chongqing, Chongqing 400320, China; ${ }^{5}$ Department of Orthopaedic Surgery, Chongqing Orthopedic Hospital of Traditional Chinese Medicine, Chongqing 400012, China; ${ }^{6}$ Department of Orthopaedic Surgery, Ningbo No. 6 Hospital, Ningbo 315040 , China Contributions: (I) Conception and design: XD Wu, W Huang; (II) Administrative support: W Huang, GX Qiu; (III) Provision of study materials or patients: XD Wu, M Tian, Y He, Y Chen, YZ Tao, W Huang; (IV) Collection and assembly of data: All authors; (V) Data analysis and interpretation: All authors; (VI) Manuscript writing: All authors; (VII) Final approval of manuscript: All authors.

Correspondence to: Wei Huang, MD, PhD. Department of Orthopaedic Surgery, The First Affiliated Hospital of Chongqing Medical University, No. 1 Youyi Road, Yuanjiagang, Yuzhong District, Chongqing 400016, China. Email: drhuangwei68@gmail.com; Gui-Xing Qiu, MD, PhD. Department of Orthopaedic Surgery, Peking Union Medical College Hospital, Chinese Academy of Medical Sciences \& Peking Union Medical College, No. 1 Shuaifuyuan Road, Wangfujing, Dongcheng District, Beijing 100730, China. Email: qiuguixing_pumch@126.com.

Background: The application of tranexamic acid (TXA) in total hip arthroplasty (THA) and total knee arthroplasty (TKA) has brought momentous changes in blood management. However, the optimal regimen of TXA has not yet been identified. This study aimed to compare the efficacy of a three-day prolongedcourse of multiple-dose of TXA with a single pre-operative dose of TXA in patients who undergo THA and TKA.

Method: We retrospectively analyzed two groups of consecutive patients who received primary unilateral THA and TKA from 2015 to 2017. One group received a three-day prolonged-course of multiple-dose of TXA, while another group received a single-dose of TXA. The primary outcomes included the changes in hemoglobin $(\mathrm{Hb})$, estimated total blood loss (TBL), and transfusion rate; the secondary outcomes included the platelet (PLT) counts, inflammatory markers, and fibrinolysis parameters.

Results: A total of 193 THA and 166 TKA procedures were included for comparison. Compared with the patients who received a single-dose of TXA, the patients who received a three-day prolonged-course of multiple-dose of TXA had smaller post-operative drops in Hb levels, which led to consistently significantly higher Hb levels in both THA and TKA. Therefore, the use of multiple-dose of TXA was associated with significantly lower maximum $\mathrm{Hb}$ drops and estimated TBL in both THA $(24.58 \pm 11.43$ vs. $30.38 \pm 11.33 \mathrm{~g} / \mathrm{L}$, $\mathrm{P}=0.001 ; 685.88 \pm 412.02$ vs. $968.94 \pm 479.9 \mathrm{~mL}, \mathrm{P}<0.0001)$ and TKA $(18.04 \pm 9.75$ vs. $27.24 \pm 10.99 \mathrm{~g} / \mathrm{L}$, $\mathrm{P}<0.0001 ; 497.35 \pm 291.03$ vs. $816.51 \pm 354.38 \mathrm{~mL}, \mathrm{P}<0.0001)$, and marginally reduced transfusion requirements (THA: 1/65 vs. 10/128; TKA: 0/70 vs. 2/96). The multiple-dose group also showed higher PLT counts, continuously reduced inflammatory responses, and significantly and durably attenuated fibrinolytic responses.

Conclusions: A three-day prolonged-course of multiple-dose of TXA was consistently effective in reducing post-operative $\mathrm{Hb}$ drops, estimated $\mathrm{TBL}$, inflammatory responses, and fibrinolytic responses, which could be recommended for clinical practice. However, these findings need to be confirmed by prospective studies.

Keywords: Total hip arthroplasty (THA); total knee arthroplasty (TKA); tranexamic acid (TXA); prolongedcourse; multiple-dose 
Submitted Nov 20, 2019. Accepted for publication Feb 14, 2020.

doi: 10.21037/atm.2020.02.99

View this article at: http://dx.doi.org/10.21037/atm.2020.02.99

\section{Introduction}

Peri-operative blood management practices for elective total hip arthroplasty (THA) and total knee arthroplasty (TKA) have been revolutionized by the application of tranexamic acid (TXA) over the past decade $(1,2)$. There is a wealth of evidence in the literature demonstrating that the routine use of TXA in total joint arthroplasty (TJA) can significantly reduce peri-operative blood loss, the transfusion rate, inflammatory responses, the post-operative swelling ratio, and the need for routine post-operative blood tests (3-11); and without an evident increase in the risk of thromboembolic events $(12,13)$.

However, although TXA has been widely used in patients undergoing TJA, little or no consensus currently exists on the optimal method of administering of TXA, and whether TXA should be administered as a single-dose or multiple-dose is still disputable. Recently published clinical guidelines developed by the AAHKS, AAOS, Hip Society, Knee Society, and ASRA strongly recommend a single-dose rather than multiple-dose of TXA (2) because researchers have found that multiple-dose of TXA is not statistically superior to a single-dose in terms of blood loss or the transfusion rate $(8,9)$. Nevertheless, the clinical significance of the additional benefits of multiple-dose of TXA, including further reductions in blood loss and maximum $\mathrm{Hb}$ drops, more controlled fibrinolysis reactions and inflammatory responses, have been neglected $(14,15)$, as the effects may not be statistically significant $(2,8,9)$.

Of note, according to previous studies, the lowest levels of hemoglobin $(\mathrm{Hb})$ in TJA patients are most often found on post-operative day (POD) 3, which indicates that postoperative hidden blood loss can last approximately three days (16-18). Therefore, prolonging the application of TXA until POD3 might offer additional benefits. Here, we proposed an administration of a three-day prolonged-course of multiple-dose of TXA, and the safety of this regimen has been well established in our previous study (19). In this study, we aimed to evaluate and validate the efficacy of this novel regimen.

\section{Methods}

\section{Study design}

We conducted a retrospective analysis of the prospectively collected data of all THA and TKA patients who were consecutively admitted to our department between 2014 and 2017. Ethical approval for this retrospective cohort study was obtained from the ethics committee of our institution. The protocol for this study was approved by our institutional review board and was registered in the International Clinical Trials Registry Platform-Chinese Clinical Trial Registry (ChiCTR1800015422). The present work was reported following the Strengthening the Reporting of Cohort Studies in Surgery (STROCSS) criteria and guidelines (20).

\section{Eligibility criteria}

Adult individuals who underwent primary unilateral THA or TKA were screened for eligibility. The specific inclusion criteria were as follows: (I) patients who received a threeday prolonged-course of multiple-dose of TXA or a preoperative single-dose of intravenous (IV) TXA as prescribed below; (II) patients with normal Hb levels or mild anemia ( $\mathrm{Hb}>90 \mathrm{~g} / \mathrm{L})$; (III) patients with normal coagulation tests and platelet (PLT) counts; (IV) patients without a personal history of venous thromboembolism (VTE) (e.g., pulmonary embolism, deep vein thrombosis, superficial vein or calf muscle vein thrombosis); and (V) patients with negative pre-operative Doppler ultrasound examination results for the lower extremities. The specific exclusion criteria were as follows: (I) patients who underwent a second primary TJA, or bilateral TJA; (II) patients with joint deformities and underwent a complicated surgical procedure or tumor-related arthroplasty; (III) patients who underwent non-elective TJA; (IV) patients with an allergy to TXA; and (V) with severe hepatic or renal dysfunction.

\section{Clinical investigations}

Patients in the single-dose group received a dose of $1.5 \mathrm{~g} \mathrm{IV}$ 


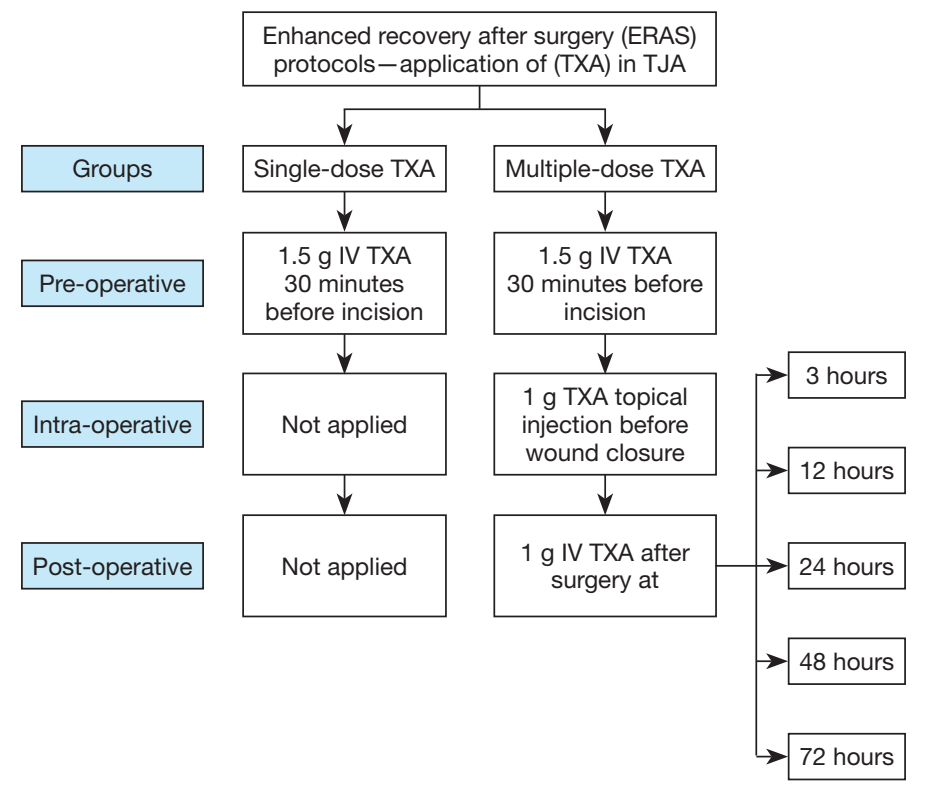

Figure 1 The formulations of TXA in the single-dose group and three-day prolonged-course of multiple-dose group. ERAS, enhanced recovery after surgery; IV intravenous; TJA, total joint arthroplasty; TXA, tranexamic acid.

TXA 30 minutes before the skin incision. For the patients in the multiple-dose group, the three-day prolonged-course of TXA was administered as follows: (I) pre-operatively, a dose of $1.5 \mathrm{~g}$ IV TXA was given 30 minutes before the skin incision; (II) intra-operatively, a dose of $1 \mathrm{~g}$ of TXA was injected intra-articularly before wound closure; and (III) post-operatively, a dose of $1 \mathrm{~g}$ IV TXA was given at 3, 12, 24, 48, 72 hours later (Figure 1). In particular, the additional post-operative multiple doses of TXA were administered post-operatively at these specific time points for the following purposes: post-operative TXA was given at 3 and 12 hours to reduce the severe bleeding caused by extensive dissections through bony and surrounding soft tissues, as well as combat hyperfibrinolysis caused by tourniquet application $(21,22)$; TXA was administered in the early post-operative rehabilitation period (at 24,48 , and 72 hours) to decrease potential wound errhysis or leakage, reduce secretion and inflammatory responses, improve the range of motion and promote early return to ambulation $(14,23,24)$.

\section{Peri-operative management}

All THA and TKA procedures were completed under general anesthesia by the same surgical team, consisting of two senior surgeons. All the THA procedures assessed in this study were performed via the posterolateral approach with the patient lying in the lateral position, while all TKA procedures were performed with the use of a tourniquet and medial parapatellar approach.

All patients followed the same enhanced recovery after surgery (ERAS) multi-modal peri-operative care pathways, including standard post-operative care, pain management, and anticoagulation therapy. The closed-suction drainage systems were placed in all TKA patients, the drainage tubes were clamped for 4 hours after TKA, and the drains were typically removed 24 hours after surgery. The indwelling urinary catheters were placed after the induction of general anesthesia and removed after 24 hours. The nerve block or patient-controlled analgesia was commonly used for postoperative analgesia, and the analgesia pump maintains analgesia for 2 to 3 days. A restrictive red blood cell transfusion strategy ( $\mathrm{Hb}<7.0 \mathrm{~g} / \mathrm{dL}$ or presenting symptomatic anemia with a $\mathrm{Hb} \geq 7.0 \mathrm{~g} / \mathrm{dL}$ ) was adopted in our institution (25). Routine laboratory tests were obtained before surgery, and 1, 3, 5, 7 days after surgery.

\section{Outcome measurements}

The safety of the three-day prolonged-course of multipledose of TXA was evaluated in a separate study (19), and this study was designed to compare the efficacy of the 
Table 1 Baseline characteristics and peri-operative demographics of the included patients

\begin{tabular}{|c|c|c|c|c|c|c|}
\hline Grouping & \multicolumn{3}{|c|}{ THA } & \multicolumn{3}{|c|}{ TKA } \\
\hline Age (y) & $65.52 \pm 14.30$ & $64.84 \pm 14.16$ & 0.754 & $68.33 \pm 7.40$ & $67.28 \pm 8.534$ & 0.411 \\
\hline Gender (male/female) & $29 / 36$ & $54 / 74$ & 0.761 & $11 / 59$ & $26 / 70$ & 0.092 \\
\hline BMI $\left(\mathrm{kg} / \mathrm{m}^{2}\right)$ & $23.49 \pm 3.26$ & $22.94 \pm 3.62$ & 0.327 & $25.66 \pm 3.34$ & $24.48 \pm 3.92$ & 0.057 \\
\hline ASA grade & $2.50 \pm 0.72$ & $2.38 \pm 0.65$ & 0.215 & $2.27 \pm 0.48$ & $2.17 \pm 0.52$ & 0.176 \\
\hline Operated side (left/right) & $29 / 36$ & $74 / 54$ & 0.094 & $36 / 34$ & $36 / 60$ & 0.083 \\
\hline Postoperative hospital stay & $8.65 \pm 6.49$ & $9.27 \pm 8.15$ & 0.595 & $8.49 \pm 7.403$ & $8.94 \pm 5.513$ & 0.653 \\
\hline
\end{tabular}

THA, total hip arthroplasty; TKA, total knee arthroplasty; TXA, tranexamic acid; ASA, American Society of Anesthesiologists.

three-day prolonged-course of multiple-dose of TXA with a single-dose of TXA. The primary outcomes included the changes in $\mathrm{Hb}$, estimated total blood loss (TBL), and transfusion rate; the secondary outcomes included the PLT counts, inflammatory markers [C-reactive protein (CRP), erythrocyte sedimentation rate (ESR)], and fibrinolysis response parameters [D-dimer (D-D), fibrinogen/fibrin degradation product (FDP)].

The TBL was calculated based on the changes in hematocrit and the estimated blood volume by the Gross and Nadel formula $(26,27)$. Specifically, if a red blood cell transfusion was performed, the estimated TBL was considered equivalent to the volume transfused plus the blood loss calculated, based on the assumption that all the red blood cell transfusions contained the same number of cells $(22,28)$.

Demographic and clinical pre-operative characteristics of the included patients were also collected, which mainly included their age, gender, height, weight, body mass index, American Society of Anesthesiologists (ASA) score, surgical diagnosis, operation time, and length of post-operative hospital stay.

\section{Statistical analysis}

The quantitative parameters were described as the means \pm standard deviations (SDs), and the qualitative parameters were expressed as frequencies or percentages. The Pearson chi-square test or Fisher's exact test was used to compare the categorical variables, and the Student's $t$-test or Wilcoxon rank-sum test was used to compare the continuous variables. All tests were two-sided, and $\mathrm{P}$ values less than 0.05 were considered statistically significant. Analyses were performed using SPSS statistical software version 21.0 software (SPSS Inc., Chicago, IL, USA).

\section{Results}

\section{Patient demographics}

A total of 193 patients who underwent THA procedures and 166 patients who underwent TKA procedures met the eligibility requirements and were included in this study. In the comparison of the baseline characteristics, we found no significant difference between the groups (Table 1). The average age was 65.07 years for the THA patients and 
Table 2 The peri-operative changes in Hb levels in primary total hip and knee arthroplasty

\begin{tabular}{|c|c|c|c|c|c|}
\hline $\mathrm{Hb}$ levels (g/L) & Pre & POD1 & POD3 & POD5 & POD7 \\
\hline Multiple-dose TXA & $123.40 \pm 14.79$ & $107.35 \pm 15.44$ & $101.14 \pm 11.63$ & $102.92 \pm 13.75$ & $106.20 \pm 10.63$ \\
\hline Single-dose TXA & $124.49 \pm 14.00$ & $105.12 \pm 14.48$ & $96.29 \pm 14.48$ & $97.40 \pm 13.55$ & $99.40 \pm 14.29$ \\
\hline$P$ value & 0.611 & 0.323 & 0.021 & 0.017 & 0.032 \\
\hline Multiple-dose TXA & $125.74 \pm 12.26$ & $115.19 \pm 12.17$ & $110.29 \pm 13.93$ & $109.95 \pm 12.39$ & $112.23 \pm 13.14$ \\
\hline Single-dose TXA & $124.43 \pm 12.83$ & $110.39 \pm 11.68$ & $99.08 \pm 12.13$ & $99.91 \pm 12.24$ & $102.24 \pm 11.90$ \\
\hline$P$ value & 0.507 & 0.011 & $<0.0001$ & $<0.0001$ & 0.001 \\
\hline
\end{tabular}

Pre, Pre-operation; POD, Post-operative day; THA, total hip arthroplasty; TKA, total knee arthroplasty; TXA, tranexamic acid; Hb, hemoglobin.
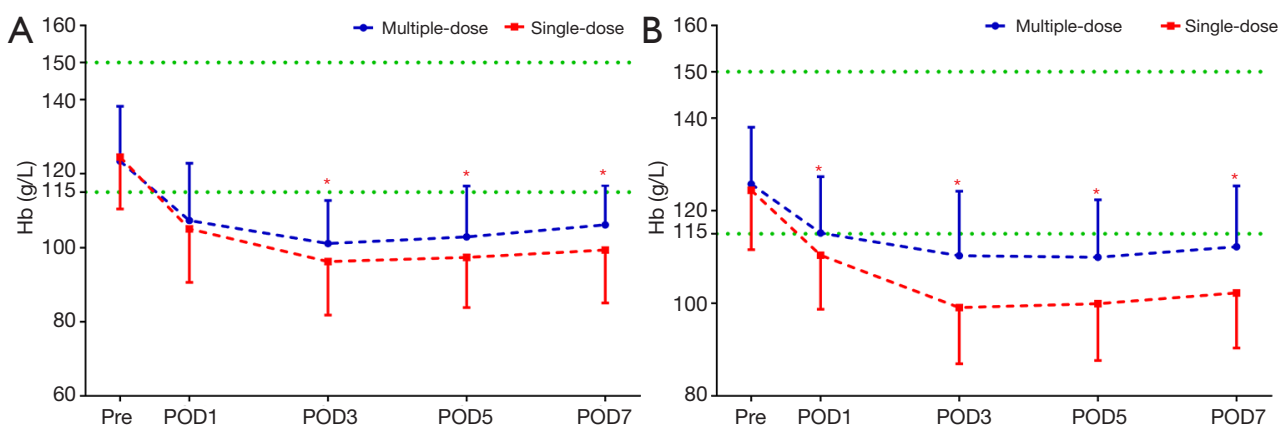

Figure 2 The peri-operative Hb levels in the THA (A) and TKA (B) patients. The asterisks $\left(^{*}\right)$ indicate values that were statistically significantly different between the three-day prolonged-course of multiple-dose and single-dose groups. The horizontal green dotted lines indicate the normal range (upper green line for the upper limit, lower green line for the lower limit). Hb, hemoglobin; THA, total hip arthroplasty; TKA, total knee arthroplasty; Pre, pre-operation; POD, post-operative day.

67.72 years for the TKA patients; $56.99 \%$ of the THA patients and $77.71 \%$ of the TKA patients were female; the majority of the patients received direct oral factor Xa inhibitor for VTE prophylaxis, and few patients received low-molecular-weight heparin.

\section{Primary efficacy outcomes}

Compared with the patients receiving a single-dose of TXA, the patients receiving the three-day prolonged-course of multiple-dose of TXA showed consistently achieved significantly higher Hb levels after THA (on POD3, POD5, POD7) and TKA (on POD1, POD3, POD5, POD7) and consistently reduced post-operative drops in $\mathrm{Hb}$ levels (Pre-POD1, POD1-POD3) after both THA (16.05 vs.
$19.37 \mathrm{~g} / \mathrm{L} ; 6.21$ vs. $8.83 \mathrm{~g} / \mathrm{L})$ and TKA (10.55 vs. $14.04 \mathrm{~g} / \mathrm{L}$; 4.9 vs. $11.31 \mathrm{~g} / \mathrm{L}$ ) (Table 2). Consequently, the maximum $\mathrm{Hb}$ drop and estimated TBL were also significantly lower in the multiple-dose group than in the single-dose group in both THA $(24.58 \pm 11.43$ vs. $30.38 \pm 11.33 \mathrm{~g} / \mathrm{L}, \mathrm{P}=0.001$; $685.88 \pm 412.02$ vs. $968.94 \pm 479.9 \mathrm{~mL}, \mathrm{P}<0.0001$;) and TKA $(18.04 \pm 9.75$ vs. $27.24 \pm 10.99 \mathrm{~g} / \mathrm{L}, \mathrm{P}<0.0001 ; 497.35 \pm 291.03$ vs. $816.51 \pm 354.38 \mathrm{~mL}, \mathrm{P}<0.0001$ ) (Figure 2). The proportion of patients who received a transfusion was also marginally lower in the multiple-dose group than in the single-dose group (THA: $1 / 65$ vs. $10 / 128, \mathrm{P}=0.148$; TKA: $0 / 70$ vs. $2 / 96, \mathrm{P}=0.509)$. This substantial and clinically significant difference should be highlighted, even though a statistically significant difference was not observed due to infrequency. 

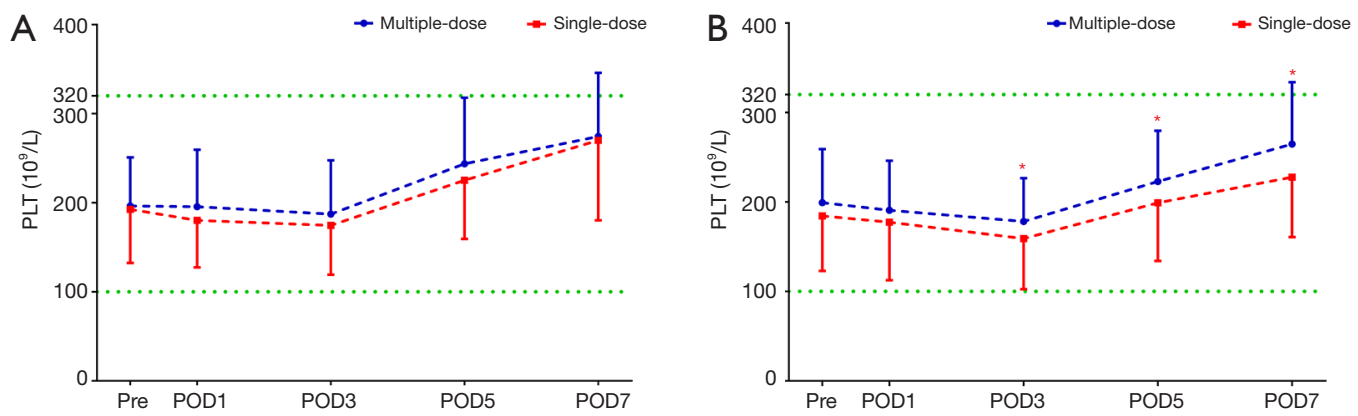

Figure 3 The peri-operative PLT counts in the THA (A) and TKA (B) patients. The asterisks $\left(^{*}\right)$ indicate values that were statistically significantly different between the three-day prolonged-course of multiple-dose and single-dose groups. The horizontal green dotted lines indicate the normal range (upper green line for the upper limit, lower green line for the lower limit). PLT, platelet; THA, total hip arthroplasty; TKA, total knee arthroplasty; Pre, pre-operation; POD, post-operative day.
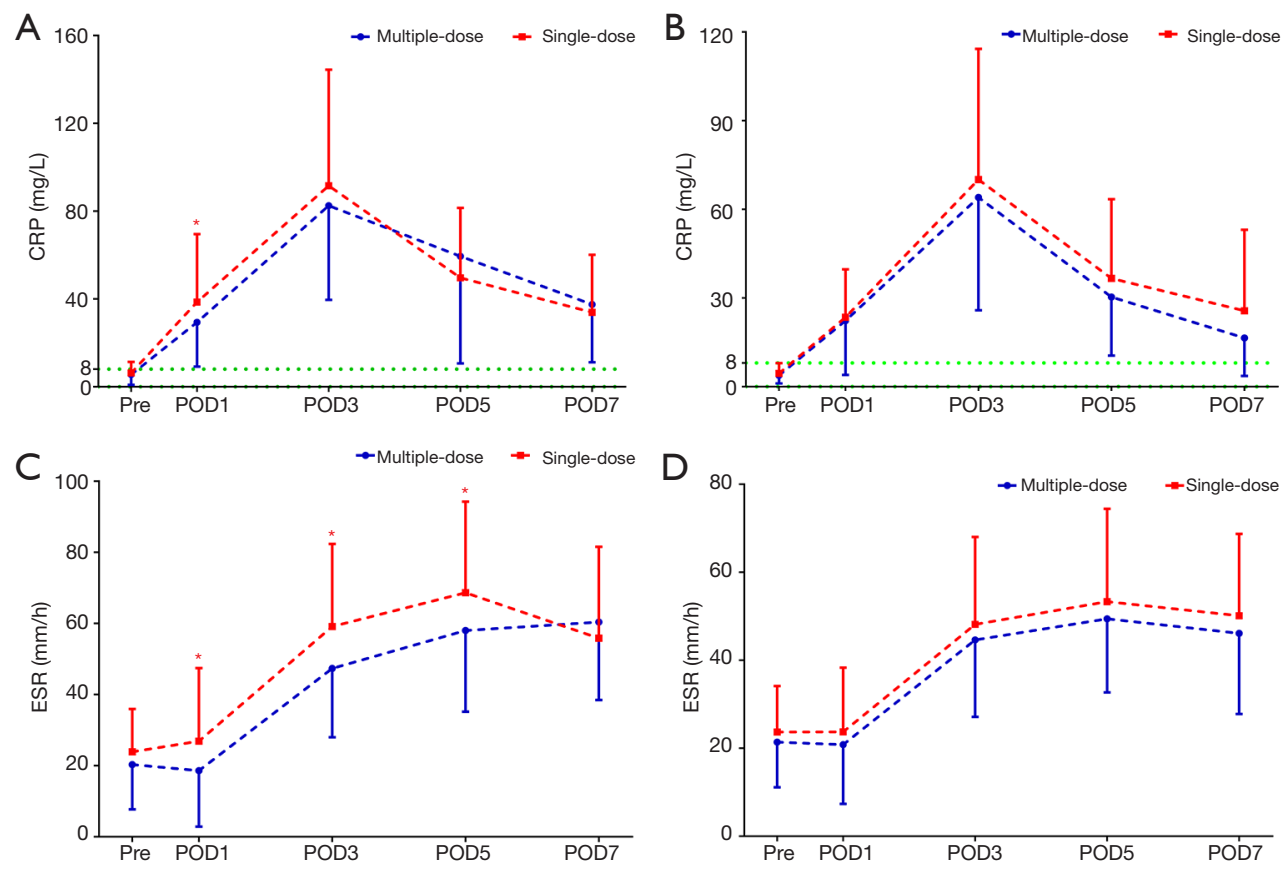

Figure 4 The peri-operative CRP and ESR in the THA (A,C) and TKA (B,D) patients. The asterisks $\left(^{*}\right)$ indicate values that were statistically significantly different between the three-day prolonged-course of multiple-dose and single-dose groups. The horizontal green dotted lines indicate the normal range (upper green line for the upper limit, lower green line for the lower limit). CRP, C-reactive protein; ESR, erythrocyte sedimentation rate; THA, total hip arthroplasty; TKA, total knee arthroplasty; Pre, pre-operation; POD, post-operative day.

\section{Secondary efficacy outcomes}

The PLT levels were simultaneously reserved with the $\mathrm{Hb}$ levels, but only the TKA patients in the multiple-dose group had significantly higher PLT counts on POD3, POD5, and POD7 (Figure 3). The acute phase reactants, CRP and ESR revealed a remarkable increase in the inflammatory response in all patients after surgery. The levels of CRP and ESR in the multiple-dose group were generally lower than those in the single-dose group. Nevertheless, statistically significant differences between the two groups were only detected in THA patients (Figure 4). In addition, in comparison with the single-dose of TXA, the prolonged-course of multipledose of TXA was associated with significantly and durably 

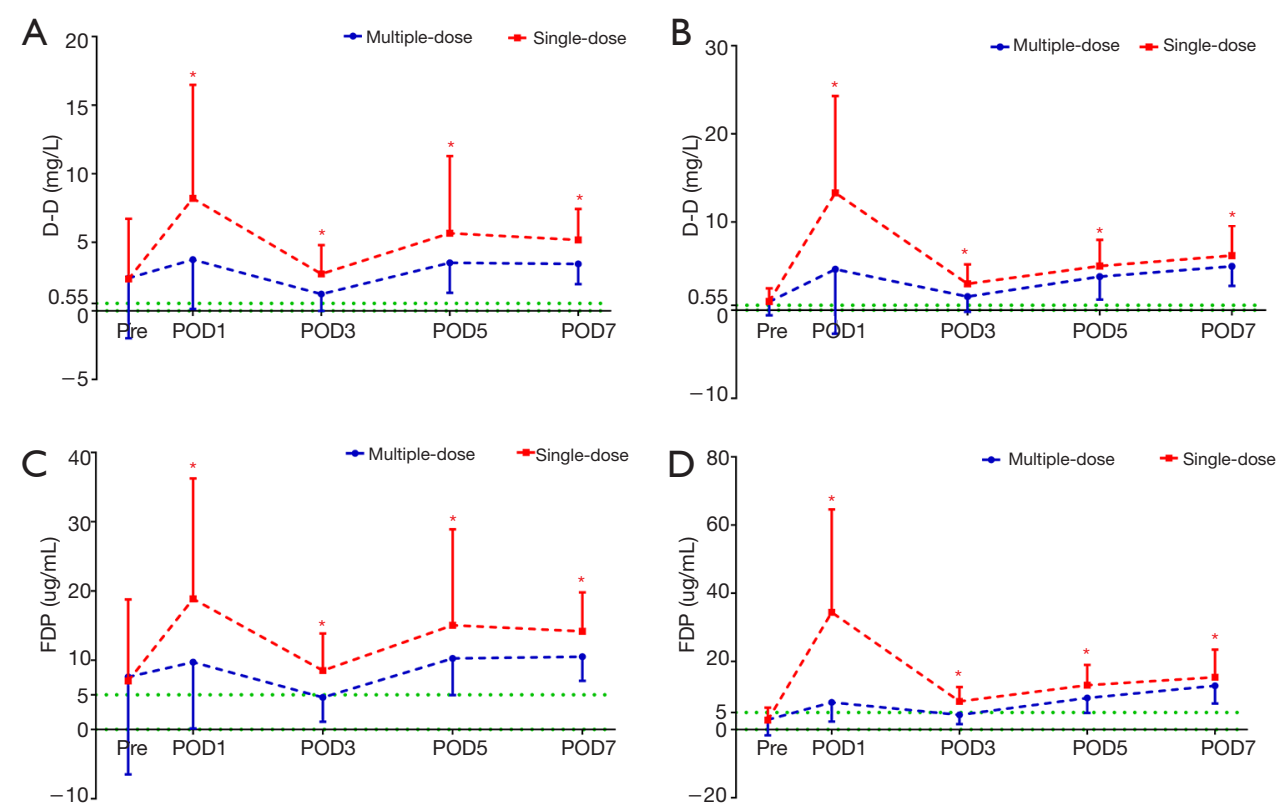

Figure 5 The peri-operative D-D and FDP in the THA (A,C) and TKA (B,D) patients. The asterisks $\left(^{*}\right)$ indicate values that were statistically significantly different between the three-day prolonged-course of multiple-dose and single-dose groups. The horizontal green dotted lines indicate the normal range (upper green line for the upper limit, lower green line for the lower limit). D-D, D-dimer; FDP, fibrinogen/fibrin degradation product; THA, total hip arthroplasty; TKA, total knee arthroplasty; Pre, pre-operation; POD, post-operative day.

reduced D-D and FDP levels $(\mathrm{P}<0.05)$ (Figure 5).

\section{Discussion}

\section{Main findings}

Our study found that: (I) compared with the patients who received a single-dose of TXA, the patients who received the three-day prolonged-course of multiple-dose of TXA had significantly and durably reduced post-operative $\mathrm{Hb}$ drops, further reduced maximum $\mathrm{Hb}$ drops and estimated TBL, marginally decreased transfusion rate; (II) multipledose of TXA was also associated with higher PLT counts, continuously reduced inflammatory response, significantly and durably attenuated fibrinolytic response.

\section{Possible mechanisms}

TXA inhibits fibrinolysis (bleeding) through the competitive inhibition of plasminogen activation. TXA almost entirely binds to plasminogen, and only $3 \%$ binds to plasma protein at therapeutic plasma levels. Therefore, the biological halflife of TXA is relatively short: the half-life is approximately 2 hours after a dose of $1 \mathrm{~g}$ IV TXA, while the biological half-life is a little longer in the joint fluid about 3 hours (29).

Previous studies have suggested that intra-operative visible blood loss is an unreliable surrogate for TBL in TJA patients, and hidden blood loss should be used to gauge the success of blood conservation methods $(30,31)$. Approximately $90 \%$ of TBL occurs during the first postoperative 24 hours $(30,32)$, which is primarily due to ongoing bleeding and hyperfibrinolysis, the latter can further increase bleeding during and immediately after surgery (30). Remarkably, the hyperfibrinolysis peaked at 6 hours after surgery and would maintain for approximately 18 hours (33). Accordingly, a single-dose of TXA is not sufficient to durably suppress fibrinolysis, and additional post-operative boluses of TXA are reasonable and justifiable. Our study found that these additional boluses of TXA (at 3, 12, 24 hours) effectively reduced the maximum $\mathrm{Hb}$ drops and estimated TBL on POD1, which is consistent with previous studies that adopted multiple-dose of TXA $(14,15)$.

Interestingly, $\mathrm{Hb}$ levels continued to decline until POD3 in TJA patients (16-18,30,32), which was often neglected in previous studies since the blood loss on POD2 and POD3 was somewhat limited when compared with the blood loss on POD1 (32). However, additional blood conservation 
measures are still worthwhile, especially for patients who experience symptoms of anemia or meeting the transfusion criteria a few days after surgery (34). Indeed, the prolongedcourse of extra doses of TXA (at 24, 48, and 72 hours) alleviated the decline in $\mathrm{Hb}$ levels. Potential explanations and mechanisms of blood loss on POD2 and POD3 in TJA patients are rarely mentioned, which might be attributed to the fibrinolysis and early post-operative exercises following surgery. Theoretically, patients who undergo TKA are encouraged to perform exercises involving isometric contractions of the quadriceps and active progressive flexion-extension of the knee from POD1, which can induce more hidden blood loss than THA. Our study did find that the $\mathrm{Hb}$ drop was more evident in TKA than in THA from POD1 to POD3 in the single-dose group, and a prolongedcourse of multiple-dose of TXA substantially slowed the decline in the $\mathrm{Hb}$ levels, especially in TKA.

Furthermore, fibrinolysis, coagulation, and inflammation systems are strongly related (35-38). Plasmin exerts proinflammatory effects by activating inflammatory cells, stimulating the production of cytokines, and other mediators $(39,40)$. TXA has anti-inflammatory effects by attenuating the formation of plasmin and regulating the inflammatory response in various surgical settings, including arthroplasty $(14,35,41-43)$. Therefore, prolonged multipledose of TXA can also alleviate the swelling and edema of the lower extremities and promote ERAS (14).

\section{Implications for clinical practice}

The recently developed clinical practice guidelines strongly recommended a single-dose rather than multiple-dose of TXA in TJA patients (2), as previous findings showed that multiple-dose of TXA failed to provide statistically superior blood management when compared with a single-dose of TXA. Thus, a single-dose of TXA is frequently sufficient, and additional doses of TXA appear to be superfluous and might result in higher drug exposures and healthcare waste $(8,9)$.

However, the clinical significance of the additional reductions in TBL (THA $283.06 \mathrm{~mL}$; TKA $319.16 \mathrm{~mL}$ ) and maximum Hb drops (THA $5.80 \mathrm{~g} / \mathrm{L}$; TKA $9.20 \mathrm{~g} / \mathrm{L}$ ), marginally decreased transfusion rates, prolonged fibrinolysis inhibition, and durably ameliorated postoperative inflammatory responses following TJA are noteworthy. More specifically, the ignored potential benefits not only include a lower risk of transfusion in patients with pre-operative anemia, alleviated the swelling of the lower extremities, a shorter length of stay, and lower morbidity in the short term, but also a lower readmission rate within 60 days of discharge and a lower risk of periprosthetic joint infection in the long term (44-46). Besides, considering that TXA has an excellent safety profile and the acquisition cost of TXA is affordable ( $\$ 2 / \mathrm{g})(19)$, a prolonged-course of multiple-dose of TXA could be recommended for clinical practice for a variety of benefits.

\section{Call for future studies}

In recent years, a growing pile of studies has investigated the application of TXA in TJA patients. However, there remains no consensus regarding the optimal regimen of TXA, including dosage and dosing frequency (multipledose, single-dose, or continuous infusion), route of administration (IV, topical/intra-articular, combined IV and topical, or oral), and timing of administration (pre-operative, intra-operative, or post-operative) (1,3-9,47-49). Although the recent guidelines suggest a pre-operative single-dose of TXA in any methods of administration can equivalently reduce blood loss and the risk of transfusion in primary TJA patients (2), our findings demonstrate that there remains an opportunity to make meaningful contributions to the clinical practice on TXA by investigating the optimal formulations of TXA for achieving the maximum reduction in blood loss in TJA patients.

\section{Strengths and limitations}

To the best of our knowledge, this is the first study that prolonged the application of TXA from pre-operative to POD3, and as many as seven doses of TXA were administered (six-dose IV-TXA, one-dose topical-TXA) for possible additional benefits; the results indicated that additional doses of TXA through POD3 are beneficial and should be recommended.

This study also had limitations. First, due to its retrospective nature, this study has several weaknesses, such as the non-randomized design, not allowing for complete control of the peri-operative variables (including demographics and co-morbidities). Therefore, these findings should be interpreted with caution, and future well-designed prospective studies are needed to confirm our findings. Second, a flat-fixed dosing regimen rather than a weight-adjusted dosing regimen of TXA was used in our department because it is potentially more convenient for both patients and clinicians. However, this dosing regimen 
might lead to a substantial difference in the concentration of TXA in the blood, and subsequently induce confounding bias to the results.

\section{Conclusions}

Our study suggests that a three-day prolonged-course of multiple-dose of TXA consistently reduced post-operative $\mathrm{Hb}$ drops and additionally reduced maximum $\mathrm{Hb}$ drops and blood loss, which were also associated with continuous benefits, including a marginally reduced transfusion rate, continuously reduced inflammatory responses, and significantly and durably attenuated fibrinolytic responses. Thus, this regimen could be recommended for clinical practice. However, these findings need further confirmation in other prospective studies.

\section{Acknowledgments}

The authors thank Cheng Chen, MD, PhD; Qiang Cheng, MD, PhD; Han Wang, MD; Heng-Kai Fan, MD; LeiLei Qin, MD; Xiao-Yu Wang, MD; Hao-Zhuo Xiao, MD; Zhang-Yu Wang, MD; Yu-Jian Li, MD; Jia-Wei Wang, MD; Yan-Ran Huang, MD. (From Department of Orthopedic Surgery, The First Affiliated Hospital of Chongqing Medical University, Chongqing, China) for their substantial contribution in acquisition of data to this work; and Yuan-Ping Jiang, MSc, (Department of Blood Transfusion, Yongchuan Hospital of Chongqing Medical University, Chongqing, China) for her substantial contribution to the interpretation of data.

Funding: This study was supported by the Special Fund for Local Scientific and Technological Development under the Guidance of the Central Government (Grant No. Z135050009017).

\section{Footnote}

Conflicts of Interest: The authors have no conflicts of interest to declare.

Ethical Statement: The authors are accountable for all aspects of the work in ensuring that questions related to the accuracy or integrity of any part of the work are appropriately investigated and resolved. The ethical approval of this study was obtained from the Ethics Committee of The First Affiliated Hospital of Chongqing Medical University (No. 20181502).
Open Access Statement: This is an Open Access article distributed in accordance with the Creative Commons Attribution-NonCommercial-NoDerivs 4.0 International License (CC BY-NC-ND 4.0), which permits the noncommercial replication and distribution of the article with the strict proviso that no changes or edits are made and the original work is properly cited (including links to both the formal publication through the relevant DOI and the license). See: https://creativecommons.org/licenses/by-nc-nd/4.0/.

\section{References}

1. Tan J, Chen H, Liu Q, et al. A meta-analysis of the effectiveness and safety of using tranexamic acid in primary unilateral total knee arthroplasty. J Surg Res 2013;184:880-7.

2. Fillingham YA, Ramkumar DB, Jevsevar DS, et al. Tranexamic acid use in total joint arthroplasty: the clinical practice guidelines endorsed by the American Association of Hip and Knee Surgeons, American Society of Regional Anesthesia and Pain Medicine, American Academy of Orthopaedic Surgeons, Hip Society, and Knee Society. J Arthroplasty 2018;33:3065-9.

3. Jain NP, Nisthane PP, Shah NA. Combined administration of systemic and topical tranexamic acid for total knee arthroplasty: can it be a better regimen and yet safe? A randomized controlled trial. J Arthroplasty 2016;31:542-7.

4. Nielsen CS, Jans Ø, Ørsnes T, et al. Combined intraarticular and intravenous tranexamic acid reduces blood loss in total knee arthroplasty: a randomized, doubleblind, placebo-controlled trial. J Bone Joint Surg Am 2016;98:835-41.

5. Shin YS, Yoon JR, Lee HN, et al. Intravenous versus topical tranexamic acid administration in primary total knee arthroplasty: a meta-analysis. Knee Surg Sports Traumatol Arthrosc 2017;25:3585-95.

6. Yuan X, Li B, Wang Q, et al. Comparison of 3 routes of administration of tranexamic acid on primary unilateral total knee arthroplasty: a prospective, randomized, controlled study. J Arthroplasty 2017;32:2738-43.

7. Yi Z, Bin $\mathrm{S}$, Jing $\mathrm{Y}$, et al. Tranexamic acid administration in primary total hip arthroplasty: a randomized controlled trial of intravenous combined with topical versus singledose intravenous administration. J Bone Joint Surg Am 2016;98:983-91.

8. Fillingham YA, Ramkumar DB, Jevsevar DS, et al. The efficacy of tranexamic acid in total knee arthroplasty: a network meta-analysis. J Arthroplasty 2018;33:3090-8.e1. 
9. Fillingham YA, Ramkumar DB, Jevsevar DS, et al. The efficacy of tranexamic acid in total hip arthroplasty: a network meta-analysis. J Arthroplasty 2018;33:3083-9.e4.

10. Halawi MJ, Plourde JM, Cote MP. Routine postoperative laboratory tests are not necessary after primary total hip arthroplasty. J Arthroplasty 2019;34:538-41.

11. Wu XD, Jiang F, Xiang BY, et al. Letter to the editor on "Routine postoperative laboratory tests are not necessary after primary total hip arthroplasty". J Arthroplasty 2019;34:1043-5.

12. Fillingham YA, Ramkumar DB, Jevsevar DS, et al. The safety of tranexamic acid in total joint arthroplasty: a direct meta-analysis. J Arthroplasty 2018;33:3070-82.e1.

13. Wu XD, Hu KJ, Sun YY, et al. Letter to the editor on "The safety of tranexamic acid in total joint arthroplasty: a direct meta-analysis". J Arthroplasty 2018;33:3365-8.e1.

14. Xie J, Hu Q, Ma J, et al. Multiple boluses of intravenous tranexamic acid to reduce hidden blood loss and the inflammatory response following enhanced-recovery primary total hip arthroplasty: a randomised clinical trial. Bone Joint J 2017;99-B:1442-9.

15. Xie J, Ma J, Yao H, et al. Multiple boluses of intravenous tranexamic acid to reduce hidden blood loss after primary total knee arthroplasty without tourniquet: a randomized clinical trial. J Arthroplasty 2016;31:2458-64.

16. Borghi B, Pignotti E, Montebugnoli M, et al. Autotransfusion in major orthopaedic surgery: experience with 1785 patients. Br J Anaesth 1997;79:662-4.

17. Ma J, Huang Z, Shen B, et al. Blood management of staged bilateral total knee arthroplasty in a single hospitalization period. J Orthop Surg Res 2014;9:116.

18. Wu YG, Zeng Y, Shen B, et al. Combination of erythropoietin and tranexamic acid in bilateral simultaneous total hip arthroplasty: a randomised, controlled trial. Hip Int 2016;26:331-7.

19. Wu XD, Chen Y, Tian M, et al. Application of thrombelastography (TEG) for safety evaluation of tranexamic acid in primary total joint arthroplasty. J Orthop Surg Res 2019;14:214.

20. Agha RA, Borrelli MR, Vella-Baldacchino M, et al. The STROCSS statement: strengthening the reporting of cohort studies in surgery. Int J Surg 2017;46:198-202.

21. Mannucci PM, Levi M. Prevention and treatment of major blood loss. N Engl J Med 2007;356:2301-11.

22. Liu X, Zhang X, Chen Y, et al. Hidden blood loss after total hip arthroplasty. J Arthroplasty 2011;26:1100-5.e1.

23. Han AS, Nairn L, Harmer AR, et al. Early rehabilitation after total knee replacement surgery: a multicenter, noninferiority, randomized clinical trial comparing a home exercise program with usual outpatient care. Arthritis Care Res (Hoboken) 2015;67:196-202.

24. Huang Z, Xie X, Li L, et al. Intravenous and topical tranexamic acid alone are superior to tourniquet use for primary total knee arthroplasty: a prospective, randomized controlled trial. J Bone Joint Surg Am 2017;99:2053-61.

25. Gu WJ, Gu XP, Wu XD, et al. Restrictive versus liberal strategy for red blood-cell transfusion: a systematic review and meta-analysis in orthopaedic patients. J Bone Joint Surg Am 2018;100:686-95.

26. Gross JB. Estimating allowable blood losscorrected for dilution. Anesthesiology 1983;58:277-80.

27. Nadler SB, Hidalgo JU, Bloch T. Prediction of blood volume in normal human adults. Surgery 1962;51:224-32.

28. Sehat KR, Evans RL, Newman JH. Hidden blood loss following hip and knee arthroplasty. Correct management of blood loss should take hidden loss into account. J Bone Joint Surg Br 2004;86:561-5.

29. FDA PI. Tranexamic Acid Injection. 2019. Available online: https://www.drugs.com/pro/tranexamic-acidinjection.html

30. Magill P, Cunningham EL, Hill JC, et al. Identifying the period of greatest blood loss after lower limb arthroplasty. Arthroplasty today 2018;4:499-504.

31. Miao K, Ni S, Zhou X, et al. Hidden blood loss and its influential factors after total hip arthroplasty. J Orthop Surg Res 2015;10:36.

32. Senthil Kumar G, Von Arx OA, Pozo JL. Rate of blood loss over 48 hours following total knee replacement. Knee 2005;12:307-9.

33. Blanié A, Bellamy L, Rhayem Y, et al. Duration of postoperative fibrinolysis after total hip or knee replacement: a laboratory follow-up study. Thromb Res 2013;131:e6-11.

34. Pedersen AB, Mehnert F, Overgaard S, et al. Allogeneic blood transfusion and prognosis following total hip replacement: a population-based follow up study. BMC Musculoskelet Disord 2009;10:167.

35. Jimenez JJ, Iribarren JL, Lorente L, et al. Tranexamic acid attenuates inflammatory response in cardiopulmonary bypass surgery through blockade of fibrinolysis: a case control study followed by a randomized double-blind controlled trial. Crit Care 2007;11:R117.

36. Esmon CT. The interactions between inflammation and coagulation. Br J Haematol 2005;131:417-30.

37. Levi M, van der Poll T. Inflammation and coagulation. Crit Care Med 2010;38:S26-34. 
38. Foley JH, Conway EM. Cross talk pathways between coagulation and inflammation. Circ Res 2016;118:1392-408.

39. Syrovets T, Lunov O, Simmet T. Plasmin as a proinflammatory cell activator. J Leukoc Biol 2012;92:509-19.

40. Levy JH. Antifibrinolytic therapy: new data and new concepts. Lancet 2010;376:3-4.

41. Shen Y, Guo Y, Mikus P, et al. Plasminogen is a key proinflammatory regulator that accelerates the healing of acute and diabetic wounds. Blood 2012;119:5879-87.

42. Ker K, Kiriya J, Perel P, et al. Avoidable mortality from giving tranexamic acid to bleeding trauma patients: an estimation based on WHO mortality data, a systematic literature review and data from the CRASH-2 trial. BMC Emerg Med 2012;12:3.

43. Morrison JJ, Dubose JJ, Rasmussen TE, et al. Military application of tranexamic acid in trauma emergency resuscitation (MATTERs) study. Arch Surg 2012;147:113-9.

44. Nagra NS, van Popta D, Whiteside S, et al. An analysis of postoperative hemoglobin levels in patients with a fractured neck of femur. Acta Orthop Traumatol Turc

Cite this article as: $\mathrm{Wu} \mathrm{XD}$, Tian $\mathrm{M}, \mathrm{He} \mathrm{Y}$, Chen $\mathrm{Y}$, Tao $\mathrm{YZ}$, Shao L, Luo C, Xiao PC, Zhu ZL, Liu JC, Huang W, Qiu GX. Efficacy of a three-day prolonged-course of multipledose versus a single-dose of tranexamic acid in total hip and knee arthroplasty. Ann Transl Med 2020;8(6):307. doi: 10.21037/atm.2020.02.99
2016;50:507-13.

45. Yazdi H, Klement MR, Hammad M, et al. Tranexamic acid is associated with reduced periprosthetic joint infection after primary total joint arthroplasty. J Arthroplasty 2020;35:840-4.

46. Wu XD, Wu D, Liu Y, et al. Letter to the editor: combined intravenous and intraarticular tranexamic acid does not offer additional benefit compared with intraarticular use alone in bilateral TKA: a randomized controlled trial. Clin Orthop Relat Res 2020. [Epub ahead of print].

47. Montroy J, Hutton B, Moodley P, et al. The efficacy and safety of topical tranexamic acid: a systematic review and meta-analysis. Transfus Med Rev 2018. [Epub ahead of print].

48. Poeran J, Rasul R, Suzuki S, et al. Tranexamic acid use and postoperative outcomes in patients undergoing total hip or knee arthroplasty in the United States: retrospective analysis of effectiveness and safety. BMJ 2014;349:g4829.

49. Zhao Z, Ma J, Ma X. Comparative efficacy and safety of different hemostatic methods in total hip arthroplasty: a network meta-analysis. J Orthop Surg Res 2019;14:3. 